\title{
The ANP Family of Peptides and Cancer Treatment
}

\author{
Michael R Kozlowski* \\ Arizona College of Optometry, Midwestern University, Glendale, Arizona, USA
}

*Corresponding author: Michael R Kozlowski, Midwestern University, 19555 North 59th Avenue, Glendale, AZ 85308, USA.

Received Date: January 29, 2021

Published Date: February 15, 2021

\begin{abstract}
Atrial natriuretic peptide (ANP) was discovered over 40 years ago, and the other members of the ANP family of peptides nearly 30 years ago. One of the effects of these peptides was found to be inhibition of the proliferation of both normal and cancer cells in vitro as well as in vivo. For the past 20 years, numerous studies have characterized the antiproliferative effects of ANP family peptides on cancer cells and proposed a role for them in the treatment of cancer. Yet, as of 2021, no anti-cancer drugs have been developed from ANP. Recently, however, events have propelled two ANPrelated agents into clinical development for treating cancer. The present mini review outlines the history of ANP, from its discovery to the recent entry of ANP-related peptides into clinical trials for cancer.
\end{abstract}

Keywords: Atrial natriuretic peptide; ANP; Small peptide; Cancer treatment; Pancreatic cancer

Abbreviations: ANP: Atrial natriuretic peptide; MAPK: Mitogen activated protein kinase; KP: Kaliuretic peptide; LANP: Long-acting atrial natriuretic peptide; VDL: Vessel dilator protein

\section{Introduction}

Atrial natriuretic peptide (ANP) was first identified in the 1950 's as the component in cardiac atrium extracts that lowered blood pressure by stimulating sodium and water excretion [1]. The peptide was subsequently isolated, purified and characterized in the 1980 's. It was determined that ANP was generated by the cleavage of a larger precursor protein, proANP. In the early 1990's, cleavage of this protein also was found to produce three other peptides that also modulated blood pressure and fluid regulation, although in divergent ways. These peptides were kaliuretic peptide (KP), longacting atrial natriuretic peptide (LANP), and vessel dilator protein (VDL) $[2,3]$. In this mini review, this group of peptides, together with ANP, will be called the ANP family of peptides, and each individual peptide referred to as an ANP family peptide. The major functions of ANP were characterized in the 1980's and 90's, and found to include diuresis, natriuresis, vasorelaxation, increased vascular permeability, and antagonism of the renin-angiotensin system. In addition to these hemodynamic effects, however, ANP was also found to inhibit cardiac hypertrophy and remodeling in response to pressure overload. This suggested that ANP may play a role in regulating cell growth and physiology. The effects of ANP on cell functioning were further investigated in several studies performed beginning in the 1990's. One of the effects identified was inhibition of cell proliferation [1]. Since unregulated cell proliferation is a major component of the pathology of cancer, such an activity points to a potential role of ANP in the treatment of cancer.

\section{Inhibition of the Proliferation of Normal Cells by} ANP

In a number of studies, beginning in the early 1990's, ANP was shown to inhibit the proliferation of a variety of types of normal cells, including rat astrocytes, mouse and rat mesangial cells, rat thymocytes, human airway smooth muscle cells, bovine aortic endothelial cells, rat gastric epithelial cells, and rat ventricular 
fibroblasts [4-15]. The concentration range for the inhibitory effects varied from nanomolar $[4,10,14]$ to micromolar $[8,9]$. The reduction in cell proliferation was not always accompanied by reduced viability, suggesting a primary effect of ANP on the generation of new cells [10]. The effectiveness of ANP against some cell types was only explored under conditions of mitogen or growth factor-stimulated proliferation $[5,8,10,12]$. In one study, inhibition of growth by ANP was only seen in the presence of a growth factor with no effect noted on basal growth [5]. This finding, along with later work, suggested that ANP may have a minimal effect on the basal growth of normal cells [16]. Certain ANP analogs (e.g., cANP423) and other peptides related to ANP (e.g., C-type natriuretic peptide) were also found to have similar effects $[7,17,18]$. Several mechanisms were proposed for the reduction of cell proliferation by ANP, including inhibition of signaling through the MAPK or Akt pathways $[12,13,19,20]$. This also pointed to a potential role of ANP in cancer treatment since deregulation of signaling in each of these pathways has been proposed as contributing to the development of cancer $[21,22]$.

\section{Inhibition of the Proliferation of Cancer Cells by ANP}

A more direct indication that ANP might be useful in cancer treatment came from examining its effects on cancer cells themselves. These studies, which began in the early 2000's, showed that ANP could reduce the proliferation of a number of types of cancer cells in vitro, including those derived from human breast cancer, human cervical cancer, human and rat colon cancer, human and mouse gastric cancer, human glioblastoma, human heart cancers, human kidney cancer, human small cell and squamous lung cancers, human and mouse melanoma, human and mouse neuroblastoma, human prostate cancer, and human thyroid cancer $[3,18-20,23,24]$. The concentration range for the antiproliferative effects extended from nanomolar to micromolar, as it did in normal cells, but in some of these studies, ANP had a non-monotonic concentration-response curve for inhibition. In one case, higher concentrations were inhibitory whereas lower concentrations stimulated cell proliferation while, in another case, lower concentrations were inhibitory whereas higher concentrations had no effect $[19,23]$. This is instructive when considering the use of ANP in the treatment of cancer, suggesting that using the correct dosage could be important for effective therapy. The effects that ANP family peptides produce on cancer cells were not seen in a number of types of normal cells that were unstimulated by growth factors or mitogens [16]. The potential use of ANP as an anticancer agent was addressed in many of these studies [3,19,20,23].

\section{Effects of ANP Family Peptides on Cancer Cells in Vitro and in Vivo}

Based on the inhibitory effects of ANP on cancer cell proliferation, Vesely and his colleagues conducted a series of studies to determine whether the other ANP family peptides had similar effects, beginning in the early 2000's. They demonstrated that KP, LANP, and VDL were also able to inhibit the proliferation of cells derived from human pancreatic, colon, prostate, breast, kidney and thyroid carcinomas; heart angiosarcoma; small cell and squamous lung cancers; and glioblastoma [3]. These effects occurred at nanomolar to micromolar concentrations, as in the studies discussed above, but the degree of inhibition of proliferation was greater than that reported those studies. The enhanced effect may have been due to a higher sensitivity to ANP family peptides in the particular types of cells used by Vesely and his colleagues since ANP was also tested in these studies and likewise produced a greater effect than it had in the earlier studies [3].

Based on these compelling results, Vesely and his colleagues also examined the effect of ANP family peptides on the growth of tumors produced by human pancreatic carcinoma, human breast carcinoma, and human small cell lung cancer in a mouse xenograft model system [25-27]. In these experiments the peptides were given either as a continuous subcutaneous infusion or as bi-weekly intravenous injections. Both types of administration appeared equally effective [28]. They found that these peptides were not only able to decrease tumor growth but, in many cases, to entirely eliminate the tumors. The reason for the magnitude of these effects may lie in the xenograft protocol used in the experiments. In their protocol, a relatively small number of cells was ingrafted [25-27] and Matrige ${ }^{\mathrm{TM}}$ was not given with the cells to provide a matrix for tumor formation. These conditions are not the most favorable for tumor development [29]. Thus, Vesely and his colleagues may have been measuring not only the inhibition of tumor growth, but also the failure of tumor establishment. If this is the case, it does not diminish the significant of these peptides as potential treatments for cancer but suggests roles in reducing both tumor growth as well as new tumor establishment (e.g., the development of metastases). More recently Nojiri and colleagues found that ANP given during lung cancer surgery reduced subsequent development of cancer metastases [30]. This was a serendipitous finding as Nojiri and his colleague were actually attempting to reduce cardiopulmonary complications arising from lung cancer surgery (e.g. chronic obstructive pulmonary disease and atrial fibrillation) by administering ANP. They found that giving patients ANP during surgery did indeed reduce these post-surgical complications [31-33]. In following up the patients, however, they also noted a reduction in the risk of developing metastases following the surgery in the patients given ANP [30].

\section{Optimization of the Antiproliferative Effects of the ANP Family Peptides}

The effectiveness of the ANP family peptides in inhibiting the proliferation of a variety of human cancer cell types both in vitro and in vivo was, at first, difficult to understand because these peptides 
share no apparent sequence homology [34]. This lack of homology made their common ability to inhibit cell proliferation hard to explain [3]. A closer examination of their sequences ultimately revealed that they all contain the same 8-amino acid length motif [34]. Subsequent work further revealed that a peptide consisting of only an optimized version of this motif (KTH-222) was more effective than an ANP family peptide containing the native motif (i.e., VDL) in decreasing the growth of human pancreatic tumors in a mouse xenograft model [34]. In this study, the percentage of inhibition of tumor growth by VDL was less than that originally reported by Vesely and colleagues [25]. This may be because the xenograft system employed in this study used a larger number of cells to seed the tumors and added Matrigel ${ }^{\mathrm{TM}}$ to the cells to provide a matrix for tumor development, thereby producing more robust tumor growth than the system used by Vesely and his colleagues [29]. Of particular significance, KTH-222 was found to be more effective in reducing the growth of human pancreatic cell tumors than the drug, gemcitabine, which is routinely used to treat pancreatic cancer in human patients [34].

\section{Conclusion}

At the time of the writing of this mini review, ANP family peptides have finally been brought to the threshold of entering clinical use in cancer by two very different paths. The first is through the discovery of a small motif found in each of the ANP family peptides that appears to underly their antiproliferative activity. KTH-222, an optimized version of this motif [34], is in the early stages of clinical development for treating pancreatic cancer. The second way is through the serendipitous finding by Nojiri and colleagues that ANP given during the perioperative period of curative lung cancer surgery reduced the recurrence of the cancer [30]. A clinical trial is now underway in lung cancer comparing surgery alone with surgery plus ANP on the development of metastases [35]. The verdict concerning the utility of ANP family peptides in the treatment of cancer will ultimately depend upon the outcome of these, and subsequent, clinical trials.

\section{Acknowledgement}

None.

\section{Conflict of Interest}

M.R. Kozlowski is a consultant for Kalos Therapeutics Inc., which is developing KTH-222.

\section{References}

1. Potter LR, Yoder AR, Flora DR, Antos LK, Dickey DM (2009) Natriuretic peptides: their structures, receptors, physiologic functions and therapeutic applications. Handb Exp Pharmacol (191): 341-366.

2. Vesely DL, Douglass MA, Dietz JR, Gower WR Jr, McCormick MT, et al. (1994) Three peptides from the atrial natriuretic factor prohormone amino terminus lower blood pressure and produce diuresis, natriuresis, and/or kaliuresis in humans. Circulation 90(3): 1129-1140.
3. Vesely DL (2013) Cardiac hormones for the treatment of cancer. Endocr Relat Cancer 20(3): R113-125.

4. Levin ER, Frank HJ (1991) Natriuretic peptides inhibit rat astroglial proliferation: mediation by C receptor. Am J Physiol 261(2 Pt 2): R453457.

5. Wolf G, Thaiss F, Schoeppe W, Stahl RA (1992) Angiotensin II-induced proliferation of cultured murine mesangial cells: inhibitory role of atrial natriuretic peptide. J Am Soc Nephrol 3(6): 1270-1278.

6. Averbukh Z, Berman S, Weissgarten J, Cohn M, Modai D (1995) Laboratory Study Atrial Natriuretic Peptide Inhibits Mesangial Cell Proliferation Induced by Early Postnephrectomy Serum. Renal Failure 17(6): 659-664.

7. Prins BA, Weber MJ, Hu RM, Pedram A, Daniels M, et al. (1996) Atrial natriuretic peptide inhibits mitogen-activated protein kinase through the clearance receptor. Potential role in the inhibition of astrocyte proliferation. J Biol Chem 271(24): 14156-14162.

8. Vollmar AM, Schmidt KN, Schulz R (1996) Natriuretic peptide receptors on rat thymocytes: inhibition of proliferation by atrial natriuretic peptide. Endocrinology 137(5): 1706-1713.

9. Segawa K, Minami K, Jimi N, Nakashima Y, Shigematsu A (1998) C-type natriuretic peptide inhibits rat mesangial cell proliferation by a phosphorylation-dependent mechanism. Naunyn Schmiedebergs Arch Pharmacol 357(1): 70-76

10. Hamad AM, Johnson SR, Knox AJ (1999) Antiproliferative effects of NO and ANP in cultured human airway smooth muscle. Am J Physiol 277(5 Pt 1): L910-918.

11. Fujisaki H, Ito H, Hirata Y, Tanaka M, Hata M, et al. (1995) Natriuretic peptides inhibit angiotensin II-induced proliferation of rat cardiac fibroblasts by blocking endothelin-1 gene expression. J Clin Invest 96(2): 1059-1065.

12. Pandey KN, Nguyen HT, Li M, Boyle JW (2000) Natriuretic peptide receptor-A negatively regulates mitogen-activated protein kinase and proliferation of mesangial cells: role of cGMP-dependent protein kinase. Biochem Biophys Res Commun 271 (2): 374-379.

13. Pedram A, Razandi M, Levin ER (2001) Natriuretic peptides suppress vascular endothelial cell growth factor signaling to angiogenesis. Endocrinology 142(4): 1578-1586.

14. Gower WR, Jr., Carter GM, McAfee Q Solivan SM (2006) Identification, regulation and anti-proliferative role of the NPR-C receptor in gastric epithelial cells. Mol Cell Biochem 293(1-2): 103-118.

15. Moubarak M, Magaud C, Saliba Y, Chatelier A, Bois P et al. (2015) Effects of atrial natriuretic peptide on rat ventricular fibroblasts during differentiation into myofibroblasts. Physiol Res 64(4): 495-503.

16. Skelton WPt, Pi GE, Vesely DL (2011) Four cardiac hormones cause death of human cancer cells but not of healthy cells. Anticancer Res 31(2): 395-402.

17. Hashim S, Li Y, Anand-Srivastava MB (2006) Small cytoplasmic domain peptides of natriuretic peptide receptor-C attenuate cell proliferation through Gialpha protein/MAP kinase/PI3-kinase/AKT pathways. Am J Physiol Heart Circ Physiol 291(6): H3144-3153.

18. Rashed HM, Sun H, Patel TB (1993) Atrial natriuretic peptide inhibits growth of hepatoblastoma (HEP G2) cells by means of activation of clearance receptors. Hepatology 17(4): 677-684.

19. Lelievre V, Pineau N, Hu Z, Ioffe Y, Byun JY, et al. (2001) Proliferative actions of natriuretic peptides on neuroblastoma cells. Involvement of guanylyl cyclase and non-guanylyl cyclase pathways. J Biol Chem 276(47): 43668-43676

20. Serafino A, Moroni N, Psaila R, Zonfrillo M, Andreola F, et al. (2012) Antiproliferative effect of atrial natriuretic peptide on colorectal cancer cells: evidence for an Akt-mediated cross-talk between NHE-1 activity and Wnt/beta-catenin signaling. Biochim Biophys Acta 1822(6): 1004-1018. 
21. Dhillon AS, Hagan S, Rath O, Kolch W (2007) MAP kinase signalling pathways in cancer. Oncogene 26(22): 3279-3290.

22. Song M, Bode AM, Dong Z, Lee MH (2019) AKT as a Therapeutic Target for Cancer. Cancer Res 79(6): 1019-1031.

23. Baldini PM, Lentini A, Mattioli P, Provenzano B, De Vito P, et al. (2006) Decrease of polyamine levels and enhancement of transglutaminase activity in selective reduction of B16-F10 melanoma cell proliferation induced by atrial natriuretic peptide. Melanoma Res 16(6): 501-507.

24. Zhang J, Zhao Z, Zu C, Hu H, Shen H, et al. (2013) Atrial natriuretic peptide modulates the proliferation of human gastric cancer cells via KCNQ1 expression. Oncol Lett 6(2): 407-414.

25. Vesely DL, Eichelbaum EJ, Sun Y, Alli AA, Vesely BA, et al. (2007) Elimination of up to $80 \%$ of human pancreatic adenocarcinomas in athymic mice by cardiac hormones. In Vivo 21(3): 445-451.

26. Vesely DL, Vesely BA, Eichelbaum EJ, Sun Y, Alli AA, et al. (2007) Four cardiac hormones eliminate up to two-thirds of human breast cancers in athymic mice. In Vivo 21(6): 973-978.

27. Villena-Heinsen C, Friedrich M, Ertan AK, Farnhammer C, Schmidt W (1998) Human ovarian cancer xenografts in nude mice: chemotherapy trials with paclitaxel, cisplatin, vinorelbine and titanocene dichloride. Anti-Cancer Drugs 9(6): 557-563.

28. Lenz A, Sun Y, Eichelbaum EJ, Skelton WPt, Pi G, et al. (2010) Twiceweekly intravenous treatment of pancreatic cancer with atrial natriuretic peptide and vessel dilator. In Vivo 24(2): 125-129.

29. Mullen P (2004) The use of Matrigel to facilitate the establishment of human cancer cell lines as xenografts. Methods Mol Med 88: 287-292.
30. Nojiri T, Hosoda H, Tokudome T, Miura K, Ishikane S, et al. (2015) Atrial natriuretic peptide prevents cancer metastasis through vascular endothelial cells. Proc Natl Acad Sci U S A 112(13): 4086-4091.

31. Nojiri T, Inoue M, Maeda H, Takeuchi Y, Sawabata N, et al. (2013) Lowdose human atrial natriuretic peptide for the prevention of postoperative cardiopulmonary complications in chronic obstructive pulmonary disease patients undergoing lung cancer surgery. Eur J Cardiothorac Surg 44(1): 98-103.

32. Nojiri T, Inoue M, Yamamoto K, Maeda H, Takeuchi Y, et al. (2012) Effects of low-dose human atrial natriuretic peptide for preventing post-operative cardiopulmonary complications in elderly patients undergoing pulmonary resection for lung cancer. Eur J Cardiothorac Surg 41(6): 1330-1334

33. Nojiri T, Yamamoto K, Maeda H, Takeuchi Y, Funakoshi Y, et al. (2012) Effect of low-dose human atrial natriuretic peptide on postoperative atrial fibrillation in patients undergoing pulmonary resection for lung cancer: a double-blind, placebo-controlled study. J Thorac Cardiovasc Surg 143(2): 488-494.

34. Kozlowski MR, Kozlowski RE (2020) A novel, small peptide with activity against human pancreatic cancer. Am J Cancer Res 10(5): 1356-1365.

35. Nojiri T, Yamamoto H, Hamasaki T, Onda K, Ohshima K, et al. (2017) Human Atrial Natriuretic Peptide for Lung Cancer Surgery G. A multicenter randomized controlled trial of surgery alone or surgery with atrial natriuretic peptide in lung cancer surgery: study protocol for a randomized controlled trial. Trials 18(1): 183. 